\title{
Recurrent Ta Low-grade Non-muscle-invasive Bladder Cancer: What Are the Options?
}

\author{
Morgan Rouprêt ${ }^{a, *}$, Per-Uno Malmstrom ${ }^{b}$, Peter Black $^{c}$

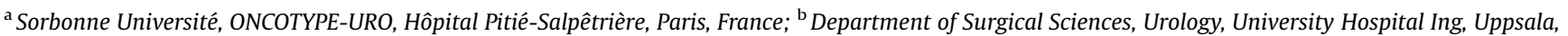 \\ Sweden; ${ }^{\mathrm{c}}$ Urologic Sciences, The University of British Columbia, Vancouver, Canada
}

\section{Article info}

Article history:

Accepted June 4, 2019

\section{Associate Editor:}

Gianluca Giannarini

\section{Keywords:}

Surveillance

Mutation

Immune checkpoint blockade

Urothelial cancer

Bladder

Neoplasm

Progression

Recurrence

\begin{abstract}
Recurrent low-grade Ta tumours, classified as intermediate-risk non-muscle-invasive bladder cancer (NMIBC), have a high risk of recurrence but a low risk of progression. This case presents a 60-yr-old female with intermediate-risk NMIBC who has been treated with sequential courses of mitomycin C followed by bacillus Calmette-Guérin (BCG). She continued to develop multiple episodes of recurrence. The discussion addresses whether the best course is repeat transurethral resection of the bladder with continued monitoring, more of the same intravesical treatments, new methods of applying these treatments, or novel treatments that might involve enrolling the patient in a clinical trial. The biggest unmet need in the field comes from the lack of a molecular marker that could help select patients for aggressive strategies.

Patient summary: Following treatment of intermediate-risk non-muscle-invasive bladder cancer with a fairly standard course of intravesical drug therapy, the patient, a relatively young woman, continued to develop recurrences of the bladder cancer. The authors discuss whether the best next course is "more of the same", device-assisted application of these treatments, or perhaps one of the new, still investigatory treatment approaches. Radical surgery (removal of the bladder) should not be necessary unless the recurrences show signs of disease progression.

(c) 2019 The Authors. Published by Elsevier B.V. on behalf of European Association of Urology. This is an open access article under the CC BY-NC-ND license (http://creativecommons.org/licenses/by-nc-nd/4.0/).

\footnotetext{
* Corresponding author. Sorbonne Université, GRC no. 5, ONCOTYPE-URO, AP-HP, Hôpital PitiéSalpêtrière, Paris F-75013, France. Tel.: +33 1421600 00; Fax: +33 142175851 . E-mail address: mroupret@gmail.com (M. Rouprêt).
}

\section{Case presentation}

A 60-yr-old female smoker presented with multiple small papillary bladder tumours discovered on a cystoscopy, located on the trigone and on the left and right bladder walls. Voided urinary cytology was negative. She underwent fluorescence-guided transurethral resection of the bladder (TURB) to optimise complete resection and decrease the recurrence rate. The diagnosis of Ta low-grade non-muscleinvasive bladder cancer (NMIBC) was confirmed by pathologic analysis. Detrusor muscle was seen in the specimen, and there was concomitant carcinoma in situ.

According to the European Association of Urology (EAU) risk-stratification guidelines [1], the patient had intermediate-risk NMIBC. According to the European Organisation for Research and Treatment of Cancer (EORTC) bladder

https://doi.org/10.1016/j.euo.2019.06.003

2588-9311/@ 2019 The Authors. Published by Elsevier B.V. on behalf of European Association of Urology. This is an open access article under the CC BY-NC-ND license (http://creativecommons.org/licenses/by-nc-nd/4.0/). 
cancer risk calculator, the probability of recurrence with this condition was $38 \%$ at $1 \mathrm{yr}$ and $62 \%$ at $5 \mathrm{yr}$, whereas the progression rate was estimated to be $1 \%$ at $1 \mathrm{yr}$ and $6 \%$ at 5 yr.

With these risk considerations in mind, therapy was initiated with eight successive weekly instillations of intravesical mitomycin $\mathrm{C}$ (MMC). The first instillation was started $5 \mathrm{wk}$ after the surgical endoscopic procedure. After $6 \mathrm{mo}$, the patient underwent another flexible cystoscopy under local anaesthesia and was diagnosed with multifocal Ta low-grade recurrence of the bladder cancer. Recurrence of this intermediate-risk NMIBC after intravesical chemotherapy was an indication to schedule a new TURB and subsequent adjuvant bacillus Calmette-Guérin (BCG) instillations after $5 \mathrm{wk}$ (optimal schedule: BCG $\times 6+$ maintenance for $1 \mathrm{yr}$ ). The patient completed the six-dose induction course of BCG, but 4 mo later she presented with a new bladder tumour recurrence.

The question is: what are the available options in recurrent Ta low-grade NMIBC after failed MMC and BCG instillations?

\section{Option A: surveillance and other intravesical therapies}

According to the EORTC risk tables [1], there was a high risk of recurrence in this case, up to $60 \%$ at $5 \mathrm{yr}$, but the risk for progression was very small (Fig. 1).

\subsection{Evidence}

\subsubsection{More of the same?}

The first question is whether the BCG instillation therapy had been optimised. This patient received only six BCG instillations and so did not fulfil the criteria for being BCG refractory; therefore, another induction course could be tried.

Some trial data [2] show that different BCG strains do not have identical antitumour activity, possibly due to different mutation patterns, and therefore this patient could also be switched to a different strain of BCG.

Finally MMC therapy can be optimised by increasing the concentration of the drug ( $40 \mathrm{mg}$ in $20 \mathrm{ml}$ ), in conjunction with urine alkalinisation and restricted fluid intake [3]. Thus, an optimised schedule could be an option if the prior therapy did not encompass this.

This is not a life-threatening disease, so one option is continued monitoring, often combined with outpatient ablation. Small, uncontrolled series with active monitoring have been reported, with a progression rate between $0 \%$ and 9\%. Soloway [4] reported that in appropriately selected patients, treatment could consist of cystoscopic surveillance and, if needed, office fulguration. This avoids or delays the surgical anaesthetic risk of TURB, thus optimising quality of life without compromising the patient's risk of cancer progression.

The patient has received two drugs so far, a chemotherapy (MMC) and an immunotherapy (BCG), and had a recurrence after each. Therefore, the question is: what next?

\subsubsection{What next?}

Other chemotherapy drugs have been found to be useful. Intravesical gemcitabine therapy was investigated in a systematic review [5] of six trials, with three having BCG as the comparator. In these trials, gemcitabine had similar efficacy in intermediate-risk cancer but was less effective in high-risk (high-grade) patients. However, the evidence is limited due to intertrial heterogeneity.



Fig. 1 - Risk of recurrence or progression of Ta low-grade NMIBC. EORTC = European Organisation for Research and Treatment of Cancer; NMIBC = nonmuscle-invasive bladder cancer.

Adapted from Sylvester et al. [1]. 
Docetaxel has been tested in phase 2 trials and found to have acceptable toxicity. One trial [6] showed a 56\% response rate in patients with BCG-refractory disease. Another study [7] reported a 59\% complete response rate in patients who had failed at least one prior course of BCG, with or without interferon; 1 - and 3-yr recurrence-free survival rates were $40 \%$ and $25 \%$, respectively.

\subsubsection{Drug combinations}

Epirubicin with interferon- $\alpha 2$ a versus BCG was used in the FinnBladder trial [8] that enrolled patients with frequently recurrent, low-grade Ta/T1 tumours. The result was that BCG was significantly more effective for avoiding recurrences, but $28 \%$ remained recurrence free after $7.4 \mathrm{yr}$ with combination therapy [8].

Combinations of gemcitabine and MMC/docetaxel have been studied as salvage therapy; the general conclusion was that approximately one-third of the patients were without recurrence after $2 \mathrm{yr}$ [9].

\subsubsection{Device-assisted intravesical therapy}

Device-assisted intravesical therapy has been used with three methods of MMC administration. The first, electromotive drug administration (EMDA), has been assessed in a recent systematic review [10] of three somewhat different randomised trials enrolling a total a 672 patients. The same principal investigator conducted all three trials. There was some uncertainty regarding occurrence of adverse events, and the quality of the evidence supporting efficacy could not be properly estimated.

The second device-assisted method is hyperthermic intravesical chemotherapy. This has also been studied in a systematic review [11] of 15 publications. The conclusion of the review was that the method was promising but the evidence is limited, due to a lack of high-quality randomised trials, and more recent randomised clinical trials with BCG as the comparator have shown divergent results.

In patients with intermediate- and high-risk bladder cancer, reduced remission-free survival at 24 mo was demonstrated in the MMC group [12], while the other group did not find a difference in patients with recurrence following induction/maintenance BCG [13].

\subsection{Conclusion and treatment recommendation}

Recurrence is a problem in this patient with recurrent lowgrade tumours, but not progression. Active monitoring with outpatient ablation is a useful alternative. Regarding secondor third-line chemotherapy, alone or in combinations, the data are promising but the evidence is limited. Both EMDA and device-assisted intravesical therapy are possible alternatives, but the data are not solid and larger trials are needed before recommending one of these approaches.

\section{Option B: novel therapy approaches}

\subsection{Evidence}

In this patient, who has already had recurrences despite two consecutive courses of treatment with MMC followed by
BCG, continuing with any additional drugs or drug combinations would be experimental treatments. If the next step is to be experimental treatment, why not do it in a trial with a novel therapy?

In cases such as this, it is important to take a step back and recognise that not all patients with intermediate-risk NMIBC require intravesical therapy [14], and even a patient who has had prior intravesical therapy may be best managed with surveillance. This is especially true for patients with infrequent, small, unifocal recurrences, who can be managed with office fulguration or TURB.

This patient, however, was having relatively rapid multifocal recurrences, and therefore some form of further therapy appeared to be necessary. Importantly, this does not mean radical cystectomy, because the risk of progression is low and therefore this is a case where the goal will be to preserve the bladder.

The first thing that this patient needs, of course, is a TURB. This should be done with blue light cystoscopy, if available, and the most recent meta-analysis [1] tells us that a single dose of chemotherapy is not appropriate at this time. However, the real question is: what are the options for adjuvant therapy? The most established option is further intravesical chemotherapy, but what other novel therapy options do we have?

\subsubsection{Novel therapies}

Several novel therapies are currently being investigated in clinical trials. These can be loosely classified as immune checkpoint inhibitors (agents that target cancer-specific mechanisms that are not necessarily the specific molecular drivers of an individual's tumour) or, more precisely, oncology (biomarker-based targeted therapies). In the current clinical trial landscape, most of these agents are being tested in high-risk BCG-unresponsive disease. This patient does not meet the high-risk criteria, but the trials are starting to move into earlier and lower-risk disease states, and this patient will likely be eligible for some exciting trials in the near future.

\subsubsection{Immune checkpoint blockade}

What about immune checkpoint blockade? These drugs target specific mechanisms of immune evasion that unleash the patient's own activated immune cells to eradicate tumour cells [15]. There are several trials targeting with CTLA4 or PD1/PD-L1, including a SWOG trial testing systemic atezolizumab in BCG-unresponsive patients [16] and a Merck trial testing pembrolizumab in the same patient population [17]. Trials are also being launched in BCG-naïve patients (eg, NCT03711032 and NCT03519256) and patients with intermediate-risk NMIBC who have received only BCG (eg, NCT03711032 and NCT03519256). There is also a phase 1 single-arm trial looking at the feasibility of delivering pembrolizumab intravesically (NCT02808143). At the present time, very little is known about targeting immune checkpoint pathways in low-grade Ta disease, and therefore it is difficult to predict whether this is going to be effective in this patient.

Single-arm phase 2 trials with single-agent immune checkpoint inhibitors are just the tip of the iceberg in the 


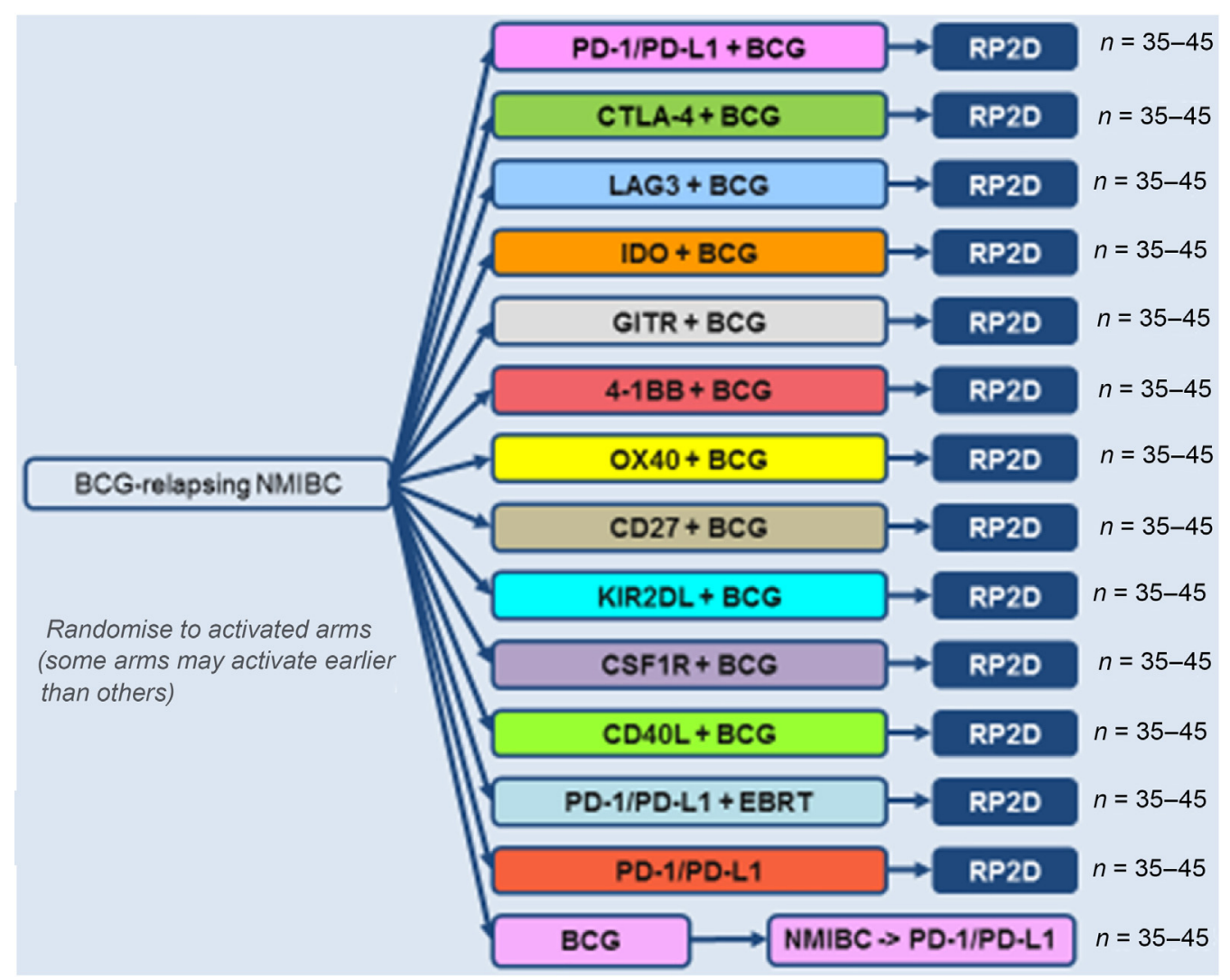

Fig. 2 - Multiphase, multiarm trial designs. BCG = bacillus Calmette-Guérin; EBRT = external beam radiotherapy; NMIBC = non-muscle-invasive bladder cancer; RP2D = recommended phase 2 dose.

Adapted from Hahn [18].

evaluation of these novel immunotherapies. Fig. 2 illustrates a trial concept proposed by Hahn [18]. The concept has evolved extensively since this figure was published (NCT03317158), but the multiarm, multiphase trial with different combinations of agents is highly attractive.

Other potential advances in immunotherapy for NMIBC include the use of alternative BCG strains [2], genetically engineered BCG strains [19], immune priming with intradermal injection [20], and alternative immune-activating agents such as the interleukin- 15 superagonist ALT803 [21].

\subsubsection{Other potential alternative new therapies}

Several interesting new drugs targeting cancer-specific mechanisms are under development, planned for trial, or already in trial.

One such agent is BC819 (BioCancell, Israel), a plasmid that is instilled into the bladder. It carries the diphtheria toxin that is under the control of an H19 promoter, which allows it to act like a Trojan horse. H19 is a transcription factor that is present only in cancer cells, so the toxin is produced strictly in cancer cells and only cancer cells are killed by it. This agent could be of particular relevance to this patient, because there is a trial planned for this agent in this disease setting (ie, intermediate-risk patients who have failed prior BCG) [22].
Oportuzumab monatox (Viventia Bio Inc., now Eleven Biotherapeutics, Inc.) is a recombinant protein consisting of a pseudomonas toxin linked to a single-chain antibody that binds EpCAM, which is expressed on the cell surface of most bladder cancer cells [23]. As with BC819, the payload (the pseudomonas toxin) is delivered specifically to the cancer cells and results in cell death.

A third alternative new therapy is Instiladrin, which is composed of a recombinant adenoviral vector that expresses interferon $\alpha 2 b$ (rAd-IFN) in combination with the excipient Syn3. This agent expresses interferon in bladder tumours when instilled into the bladder, resulting in tumour cell death. Both Instiladrin (NCT02773849) and oportuzumab monatox (NCT02449239) are currently being tested in phase 3 trials after completing successful phase 2 trials in BCG-unresponsive patients [24].

\subsection{4. "Precision oncology"}

Can molecular profiling of intermediate-risk NMIBC be used to guide the next step in treatment? There is a high likelihood that this patient's recurrent low-grade Ta tumours harbour an activating FGFR3 mutation [25] in the context of an otherwise relatively stable genome [26].

Until recently, FGFR3 inhibitors have shown disappointing results in clinical trials in the metastatic setting. 
However, recent encouraging results with a pan-FGFR inhibitor, erdafitinib, in a phase 2 trial [27] suggest that this may have been due in part to poorly effective drugs. Furthermore, FGFR3 is more likely to be driving tumour growth in a low-grade papillary tumour than in a patient with metastatic urothelial carcinoma. Multiple potent FGFR3 inhibitors are currently undergoing clinical development, and it will be critical to test these drugs in patients such as the present one.

\section{Discussion of treatment options}

In NMIBC, treatment should be based on a patient's prognosis. In order to predict, separately, the short- and long-term risks of disease recurrence and progression in individual patients, the EORTC Genito-Urinary Cancer Group has developed a scoring system and risk tables, as mentioned in the EAU guidelines [28].

The prognosis of intermediate-risk patients treated with chemotherapy has been calculated in a recently published paper [29], taking into account the history of recurrences, history of intravesical treatment, tumour grade, number of tumours, and adjuvant chemotherapy. These risk stratification and prognostic models enable outcome comparisons and standardisation of treatment and follow-up. At present, none of the models reflects current standards of treatment. As NMIBC is a heterogeneous disease, the EORTC risk tables and CUETO scoring model should be updated with previously unavailable data and recalculated [30]. A risk-based therapeutic approach is certainly recommended, but the prognostic value of pathologic factors is imperfect. Clearly in this patient, the risk of recurrence is high, but the risk of progression is very low.

The concept of active surveillance is quite new in the field of NMIBC, but to date, it has been applied mainly in low-risk tumours and/or in elderly patients [31].

Current protocols of immunotherapy are on-going and recruiting patients, but are more focused on high-risk BCGrefractory patients (eg, NCT02844816 [32] and MK-3475 [30]) or high-risk patients naïve of chemotherapy (such as POTOMAC) [33].

Further trials will be proposed soon, as the field is moving forward rapidly and these should be of interest for this patient.

The biggest unmet need for patients like the one discussed here is the lack of molecular markers to guide therapy by providing useful information on the risk of recurrence and progression, and response to treatment. In the current era of molecular medicine and advances in technologies for the molecular interrogation of biospecimens, promising new markers have been identified based on next-generation sequencing and other high-throughput profiling, such as epigenetic methylation profiling or a mini-chromosome protein complex [34-37]. More research is needed to determine the role of molecular markers in improving the predictive accuracy of currently available risk tables. New markers may identify patients with intermediate-risk bladder cancer who are likely to progress and thus deserve aggressive management, instead of endoscopic ablation and active surveillance.

\section{Summary and final treatment recommendation}

In conclusion, this patient appears to be destined to fail further intravesical chemotherapy or BCG therapy. Until new-generation molecular markers are available to identify patients who are likely to evolve rapidly, priority should be given to enrolling patients such as this one in clinical trials of the many promising novel therapies, provided that they fulfil the inclusion criteria. This could be the best way to both help this patient and learn more about bladder cancer pathways. However, in the absence of available clinical trials, observation (with periodic office or local anaesthetic fulguration) or enhanced intravesical chemotherapy remains an option. Radical cystectomy is not indicated from the perspective of preventing cancer-related deaths, yet sometimes might be needed if the patient develops a crippled bladder from all the repeated interventions.

Author contributions: Morgan Rouprêt had full access to all the data in the study and takes responsibility for the integrity of the data and the accuracy of the data analysis.

Study concept and design: Rouprêt, Malmstrom, Black.

Acquisition of data: Rouprêt, Malmstrom, Black.

Analysis and interpretation of data: Rouprêt, Malmstrom, Black.

Drafting of the manuscript: Rouprêt, Malmstrom, Black.

Critical revision of the manuscript for important intellectual content: Rouprêt, Malmstrom, Black.

Statistical analysis: None.

Obtaining funding: None.

Administrative, technical, or material support: None.

Supervision: Rouprêt, Malmstrom, Black.

Other: None.

Financial disclosures: Morgan Rouprêt certifies that all conflicts of interest, including specific financial interests and relationships and affiliations relevant to the subject matter or materials discussed in the manuscript (eg, employment/affiliation, grants or funding, consultancies, honoraria, stock ownership or options, expert testimony, royalties, or patents filed, received, or pending), are the following: Morgan Rouprêt and Per-Uno Malmstrom report no relevant personal financial relationships. Peter Black reports paid consultancies with Sitka, Merck, Roche/ Genentech, BMS, Astrazeneca, Biosyent, and Janssen.

Funding/Support and role of the sponsor: None.

\section{Acknowledgements}

Origin of Academic Content: This article has been developed from an interactive, multi-faculty scientific session presented during the 33rd Annual EAU Congress in Copenhagen, March 2018, with acknowledgment to the Co-chairs of the session: H. Barton Grossman, MD; Ashish Kamat, MD; and Arnulf Stenzl, MD, PhD. Acknowledgement is also given to Dr. Grossman and Dr. Kamat for their development of the multifaculty, interactive format of this article, and for their editorial review of the draft manuscript for submission to European Urology Oncology.

Open Access: Cepheid ${ }^{\mathbb{R}}$ provided funding to allow Open Access for this article. 


\section{References}

[1] Sylvester RJ, van der Meijden APM, Oosterlinck W, et al. Predicting recurrence and progression in individual patients with stage Ta T1 bladder cancer using EORTC risk tables: a combined analysis of 2596 patients from seven EORTC trials. Eur Urol 2006;49:466-77.

[2] Rentsch CA, Birkhauser FD, Biot C, et al. Bacillus Calmette-Guerin strain differences have an impact on clinical outcome in bladder cancer immunotherapy. Eur Urol 2014;66:677-88.

[3] Au JL, Badalament RA, Wientjes MG, et al. Methods to improve efficacy of intravesical mitomycin C: results of a randomized phase III trial. JNCI 2001;93:597-604.

[4] Soloway MS. Re: Overdiagnosis and overtreatment in cancer: an opportunity for improvement. Eur Urol 2014;65:249-50.

[5] Shelley MD, Jones G, Cleves A, Wilt TJ, Mason MD, Kynaston HG Intravesical gemcitabine therapy for non-muscle invasive bladder cancer (NMIBC): a systematic review. BJU Int 2012;109:496-505.

[6] McKieran JM, Masson P, Murphy AM, et al. Phase I trial of intravesical docetaxel in the management of superficial bladder cancer refractory to standard intravesical therapy. J Clin Oncol 2006;24:3075-80.

[7] Barlow LJ, McKiernan JM, Benson MC. Long-term survival outcomes with intravesical docetaxel for recurrent nonmuscle invasive bladder cancer after previous bacillus Calmette-Guérin therapy. J Urol 2013;189:834-9.

[8] Marttila T, Järvinen R, Liukkonen T. Intravesical bacillus CalmetteGuérin versus combination of epirubicin and interferon- $\alpha 2 \mathrm{a}$ in reducing recurrence of non-muscle-invasive bladder carcinoma: FinnBladder-6 study. Eur Urol 2016;70:341-7.

[9] Velaer KN, Steinberg RL, Thomas LJ, O'Donnell MA, Nepple KG. Experience with sequential intravesical gemcitabine and docetaxel as salvage therapy for non-muscle invasive bladder cancer. Curr Urol Rep 2016;17:38.

[10] Jung JH, Gudeloglu A, Kiziloz H, et al. Intravesical electromotive drug administration for non-muscle invasive bladder cancer. Cochrane Database Syst Rev 2017;9:CD011864.

[11] Longo TA, Gopalakrishna A, Tsivian M, et al. A systematic review of regional hyperthermia therapy in bladder cancer. Int J Hyperthermia 2016;32:381-9.

[12] Arends TJ, Nativ O, Maffezzini M, et al. Results of a randomised controlled trial comparing intravesical chemohyperthermia with mitomycin C versus bacillus Calmette-Guerin for adjuvant treatment of patients with intermediate- and high-risk non-muscleinvasive bladder cancer. Eur Urol 2016;69:1046-52.

[13] Tan WS, Panchal A, Buckley L, et al. Radiofrequency-induced thermo-chemotherapy effect versus a second course of bacillus Calmette-Guérin or institutional standard in patients with recurrence of non-muscle-invasive bladder cancer following induction or maintenance bacillus Calmette-Guérin therapy (HYMN): a phase III, open-label, randomised controlled trial. Eur Urol 2019;75:63-71.

[14] Kamat AM, Witjes JA, Brausi M, et al. Defining and treating the spectrum of intermediate risk nonmuscle invasive bladder cancer. J Urol 2014;192:305-15.

[15] Singh S, Black P. Emerging role of checkpoint inhibition in localized bladder cancer. Urol Oncol 2016;34:548-55.

[16] Singh P, Tangen C, Lerner SP, et al. S1605: phase II trial of atezolizumab in BCG-unresponsive non-muscle invasive bladder cancer. 2017. Abstract at ASCO2018. TPS4591. https://ascopubs.org/doi/abs/ 10.1200/JCO.35.15_suppl

[17] Kamat AM. KEYNOTE-057: phase-2 study of pembrolizumab for patients with BCG-unresponsive, high-risk non-muscle-invasive bladder cancer (NMIBC). J Clin Oncol 2016;34(Suppl.):TPS4576.

[18] Hahn N. ADAPT-BLADDER: modern immunotherapy in BCG-relapsing urothelial carcinoma of the bladder. NCT03317158. ClinicalTrials.gov.
[19] Nieuwenhuizen NE, Kulkarni PS, Shaligram U, et al. The recombinant bacille Calmette-Guérin vaccine VPM1002: ready for clinical efficacy testing. Front Immunol 2017;19:1147.

[20] Svatek RS, Tangen C, Delacroix S, Lowrance W, Lerner SP. Background and Update for S1602 "A Phase III randomized trial to evaluate the influence of BCG strain differences and $\mathrm{T}$ cell priming with intradermal BCG before intravesical therapy for BCG-naïve high-grade non-muscle-invasive bladder cancer. Eur Urol Focus 2018;4:522-4.

[21] Gomes-Giacoia E, Miyake M, Goodison S, et al. Intravesical ALT-803 and BCG treatment reduces tumor burden in a carcinogen induced bladder cancer rat model; a role for cytokine production and NK cell expansion. PLoS One 2014;9:e96705.

[22] BioCanCell. BioCanCell presents final data from its phase 2 study of BC-819 for early stage bladder cancer at the 2018 ASCO genitourinary cancers symposium. BioCanCell press release 06.10.18. http:// www.biocancell.com/biocancell-presents-final-data-from-itsphase-2-study-of-bc-819-for-early-stage-bladder-cancer-atthe-2018-asco-genitourinary-cancers-symposium/.

[23] Kowalski M, Guindon J, Brazas L, et al. A phase II study of oportuzumab monatox: an immunotoxin therapy for patients with noninvasive urothelial carcinoma in situ previously treated with bacillus Calmette-Guérin. J Urol 2012;188:1712-8.

[24] Sidaway P. Bladder cancer: IFN $\alpha-2 b$ gene transfer: a new approach for BCG-resistant disease. Nat Rev Urol 2017;14:583.

[25] Bakkar AA, Wallerand H, Radvanyi F, et al. FGFR3 and TP53 gene mutations define two distinct pathways in urothelial cell carcinoma of the bladder. Cancer Res 2003;63:8108-12.

[26] Hurst CD, Alder O, Platt FM, et al. Genomic subtypes of noninvasive bladder cancer with distinct metabolic profile and female gender bias in KDM6A mutation frequency. Cancer Cell 2017;32:701-5, e7.

[27] Loriot Y, Necchi A, Park SH, et al. Erdafitinib (ERDA; JNJ-42756493), a pan-fibroblast growth factor receptor (FGFR) inhibitor, in patients (pts) with metastatic or unresectable urothelial carcinoma (mUC) and FGFR alterations (FGFRa): Phase 2 continuous versus intermittent dosing. J Clin Oncol 2018;36(6 Suppl.):411.

[28] Babjuk M, Böhle A, Burger M, et al. EAU guidelines on non-muscleinvasive urothelial carcinoma of the bladder: update 2016. Eur Urol 2017;71:447-61.

[29] Lammers RJ, Hendriks JC, Rodriguez Faba OR, Witjes WP, Palou J, Witjes JA. Prediction model for recurrence probabilities after intravesical chemotherapy in patients with intermediate-risk non-muscle-invasive bladder cancer, including external validation. World J Urol 2016;34:173-80.

[30] Soukup V, Čapoun O, Cohen D, et al. Risk stratification tools and prognostic models in non-muscle-invasive bladder cancer: a critical assessment from the European Association of Urology non-muscleinvasive bladder cancer guidelines panel. Eur Urol Focus. In press. https://doi.org/10.1016/j.euf.2018.11.005.

[31] Soloway MS. Bladder cancer: active surveillance for low-grade Ta bladder tumours. Nat Rev Urol 2016;13:303-4.

[32] Study of pembrolizumab (MK-3475) in participants with high risk non-muscle invasive bladder cancer (MK-3475-057/KEYNOTE057). https://clinicaltrials.gov/ct2/show/NCT02625961.

[33] Assessment of efficacy and safety of durvalumab plus BCG compared to the standard therapy with BCG in non-muscle invasive bladder cancer (POTOMAC). https://clinicaltrials.gov/ct2/show/ NCT03528694.

[34] Roperch JP, Grandchamp B, Desgrandchamps F, et al. Promoter hypermethylation of HS3ST2 SEPTIN9 and SLIT2 combined with FGFR3 mutations as a sensitive/specific urinary assay for diagnosis and surveillance in patients with low or high-risk non-muscleinvasive bladder cancer. BMC Cancer 2016;16:704. 
[35] Witjes AJ, Morote J, Cornel EB, et al. Performance of the bladder EpiCheck $^{\mathrm{TM}}$ methylation test for patients under surveillance for non-muscle-invasive bladder cancer: results of a multicenter, prospective, blinded clinical trial. Eur Urol Oncol 2018;1:307-13.

[36] Schmitz-Drager BJ, Todenhöfer T, van Rhijn B, et al. Considerations on the use of urine markers in the management of patients with low-/intermediate-risk non-muscle invasive bladder cancer. Urol Oncol 2014;32:1061-8.

[37] Larré S, Camparo P, Comperat E, et al. Diagnostic, staging, and grading of urothelial carcinomas from urine: performance of BCA-1, a mini-array comparative genomic hybridisation-based test. Eur Urol 2011;59:250-7. 